\title{
DUALITY FOR DISTRIBUTIVE BISEMILATTICES
}

\author{
GERHARD GIERZ and ANNA ROMANOWSKA
}

(Received 9 October 1989; revised 7 March 1990)

Communicated by P. G. Trotter

\begin{abstract}
We establish a duality between distributive bisemilattices and certain compact left normal bands. The main technique in the proof utilizes the idea of Plonka sums.

1980 Mathematics subject classification (Amer. Math. Soc.) (1985 Revision): 05 F 05, 20 M 10, $08 \mathrm{C} 05,22$ A 30.
\end{abstract}

\section{Introduction}

Dualities for partially ordered structures are well known: there are Stone's dualities for Boolean algebras and distributive lattices [24, 25], there is Priestley's duality for distributive lattices $[18,19]$, and we have a Pontryagin duality for semilattices [8], to name just a few of them. All those dualities are constructed by the same method: one picks an object $P$ that "lives" in both categories (that is, the category in question and its dual), and then utilizes the contravariant hom-functors $\operatorname{Hom}(-, P)$ in order to establish dualities between the two categories. This method of constructing dualities was discussed extensively in [7] and [3].

In this paper, we shall establish a duality between distributive bisemilattices on one side and compact totally disconnected partially ordered left normal bands on the other side. This duality presents no exception in its general nature: the functors between the two categories are lifted hom-functors, and the object $P$ that "lives" in both categories is a certain subdirectly irreducible distributive bisemilattice with three elements. This duality will be a

(c) 1991 Australian Mathematical Society 0263-6115/91\$A2.00+0.00 
common generalization of Priestley's duality for distributive lattices and the Pontryagin duality for semilattices. However, although this duality might be interesting by itself and there are some intriguing algebraic manipulations in the proofs, the main point in this paper lies in the fact that for the first time we use the technique of Plonka sums in duality theory. The objects of both categories in question (the category of distributive bisemilattices and the category of left normal bands) allow a representation of Płonka sums of certain "simple" objects, and in some sense the duality works by just dualizing those Plonka sums. On the side of the category of compact totally disconnected left normal bands, one would need a topological analog of Plonka sums. Since at this point the tools of "continuous Plonka sums" are not yet available, we cannot make this dualization of Plonka sums completely explicit. But this paper could serve as a first indication on how continuous Plonka sums should be defined and on how they can be used in duality theory. Moreover, almost identical arguments to the one used in this note should lead to dualities between Płonka sums of Boolean algebras on one side and Płonka sums of sets (that is, left normal bands) on the other side. There also might be a way to lift the duality for locally compact abelian groups to a duality of "continuous Płonka sums" of locally compact abelian groups.

Before we formulate our duality theory, we have to discuss some basic facts on distributive bisemilattices. This will be done in Section 2. The objects of the dual category for distributive bisemilattices are some topological partially ordered algebras. In Section 3 we introduce partially ordered left normal bands, investigate their properties, and give a structure theorem. The dual objects for bisemilattices will be based upon these partially ordered left normal bands. Since partially ordered left normal bands are in fact bi-ordered (that is, they support two canonical partial orders), we will need some properties on order preserving maps between bi-ordered sets. This information will be compiled in Section 5. In Section 6, topological partially ordered left normal bands are discussed, and a structure theorem for those objects is given. In Section 7, we finally formulate and prove our duality theorem. here we show that the category of distributive bisemillatices is dually equivalent to the category of compact totally disconnected partially ordered left normal bands.

We recommend Nachbin's book [13] for properties of topological partially ordered sets. Basic facts on algebraic semilattices are found in [8], and we refer to Mac Lane's book [12] for definitions and results in category theory. A standard reference for lattices, semilattices, and partially ordered sets in Birkhoff's book [2].

We also would like to express our thanks to the referees of this paper. Their numerous valuable comments helped to improve this paper considerably. 


\section{Distributive bisemilattices and left normal bands}

In this paper $(S, \leq)$ is called a partially ordered set if the relation $\leq$ is reflexive, antisymmetric and transitive (thus a poset in the meaning of [2]; see also [4]).

A semilattice $(S, \cdot)$ is a commutative idempotent semigroup. If $(S, \cdot)$ is a semilattice, then we can define a partial order $\leq$ on $S$ by setting $x \leq y$ if and only if $x \cdot y=x$. If $x, y \in S$ are given, then $x \cdot y$ is the largest lower bound of $x$ and $y$ with respect to the partial order $\leq$, and we say that $(S, \leq)$ is a meet-semilattice. Similarly, if $(S,+)$ is a semilattice, then we can define a partial order $\leq_{+}$by $x \leq_{+} y$ if and only if $x+y=y$. In this case, $x+y$ is the least upper bound of the elements $x, y \in S$, and we say that $\left(S, \leq_{+}\right)$is a join semilattice.

A set $S$ with two semilattice operations + and - is called a bisemilattice. One regards $(S, \leq)$ as a meet-semilattice (and refers to a "meet") and $\left(S, \leq_{+}\right)$as a join-semilattice (+ is the "join"). Simple examples are furnished by lattices $(L, \vee, \wedge)$ with the usual join and meet operations (for which the two partial orders $\leq_{\vee}$ and $\leq_{\Lambda}$ coincide with the usual partial order), and the bisemilattice $(S, \cdot, \cdot)$ obtained form a semilattice $(S, \cdot)$ by taking the same underlying set $S$ with the semilattice operation considered twice, once as meet operation and once as join. If $w$ is a bisemilattice word without parentheses, then we make the convention that the operation - is carried out before the operation + .

A bisemilattice $(S, \cdot,+)$ satisfying the equations

$$
x \cdot(y+z)=x \cdot y+x \cdot z, \quad x+y \cdot z=(x+y) \cdot(x+z)
$$

is called a distributive bisemilattice (see [15] and [17], where distributive bisemilattices are investigated under the name of distributive quasilattice, and [22]). Examples for distributive bisemilattices are distributive lattices and the bisemilattices of the form $(S, \cdot, \cdot)$ obtained from a semilattice $(S, \cdot)$.

\section{For later use we need}

2.1. Lemma [15]. If $(S, \cdot,+)$ is a distributive bisemilattice, then for all $x, y, z \in S$ we have $x+y \cdot z=x+y \cdot z+x \cdot z$.

An idempotent semigroup $(A, \star)$ satisfying the equation $x \star y \star z=x \star z \star y$ is called a left normal band. Examples of left normal bands are semilattices and left-zero bands, that is, semigroups satisfying the equation $x \star y=x$. Every left normal band may be decomposed as a Plonka sum into the disjoint union of its maximal left-zero subbands according to the following theorem (cf. [9], [15], [22]). 
2.2. TheOREM. Let $(A, \star)$ be a left normal band. Then

$$
(x, y) \in \Theta_{A} \Leftrightarrow x \star y=x \text { and } y \star x=y
$$

defines a congruence relation $\boldsymbol{\Theta}_{A}$ on $(A, \star)$ such that $(I, \star)=\left(A / \boldsymbol{\Theta}_{A}, \star\right)$ is the largest commutative quotient of $(A, \star)$ and hence a semilattice. For each $i \in I$ the corresponding congruence class $A_{i}$, also called a Ptonka fibre of $(A, \star)$, is a maximal left-zero subband of $(A, \star)$. Clearly, $A$ is the disjoint union of these fibres. Moreover, for $i, j \in I$ with $i \geq j$ (that is, $i \star j=j$ ) the so called Plonka maps $p_{i j}: A_{i} \rightarrow A_{j}$ are defined by $x \mapsto x \star a_{j}$ for some $a_{j} \in A_{j}$, where $x \star a_{j}=x \star b_{j} \in A_{j}$ holds for all $x \in A$ and all $a_{j}, b_{j} \in A_{j}$. These maps are homomorphisms satisfying

(a) $p_{i, i}=\mathrm{id}_{A_{i}}$ and $p_{i, j} \circ p_{j, k}=p_{i, k}$ for $i \geq j \geq k$.

Together with the operations on $\left(A_{i}, \star\right)$ and on $(I, \star)$, they determine the operation * on $A$ by

(b) $a_{i} \star b_{j}=a_{i} p_{i, i \star j} \star b_{j} b_{j, i \star j}=a_{i} p_{i, i \star j}$.

Conversely, let $(I, \star)$ be a semilattice, $A$ the disjoint union of left-zero bands $\left(A_{i}, \star\right)$ for $i \in I$ and $p_{i, j}$ a set of homomorphisms satisfying $(a)$. Then $(b)$ defines an operation on $A$ such that $(A, \star)$ is a left normal band.

\section{Ordered left normal bands}

3.1. Lemma [15]. Let $(S, \cdot,+)$ be a distributive bisemilattice and define an operation $\star$ on $S$ by

$$
x \star y=x+x \cdot y=x \cdot(x+y) .
$$

Then $(S, \star)$ is a left normal band.

3.2. Proposition [16]. If $(S, \cdot,+)$ is a distributive bisemilattice, then

$$
\begin{gathered}
(a+c) \star(b+d)=(a \star b)+(c \star d), \\
(a \cdot c) \star(b \cdot d)=(a \star b) \cdot(c \star d),
\end{gathered}
$$

That is, the algebras $(S, \star,+)$ and $(S, \star, \cdot)$ are entropic.

3.3. Proposition. If $(S, \cdot,+)$ is a distributive bisemilattice, then

(i) $(S, \star)$ is commutative (that is, a semilattice) if and only if $x \cdot y=x+y$ for all $x, y \in S$,

(ii) $(S, \star)$ satisfies the equation $x=x \star y$ if and only if $(S, \cdot,+)$ is a distributive lattice.

Proof. (i) If $x \cdot y=x+y$, then $x \star y=x+x \cdot y=x \cdot x \cdot y=x \cdot y=$ $y \cdot x=y \star x$. Conversely, assume that $\star$ is commutative. Then for all 
$x, y \in S$ we have $x+x \cdot y=y+x \cdot y$. Multiplying this equation by $x$ yields $x+x \cdot y=x \cdot y+x \cdot y=x \cdot y$. Using both equations, we obtain

$$
x+y=(x+y) \cdot(x+y)=x+x \cdot y+y+x \cdot y=x+x \cdot y=x \cdot y .
$$

(ii) If $(S, \cdot,+)$ is a distributive lattice, then $x \star y=x+x \cdot y=x$. Conversely, if $(S, \star)$ satisfies this equation, then $(S, \cdot,+)$ satisfies the absorption laws, and therefore is a lattice.

In addition to the operation $\star$, there are the two partial orders $\leq_{+}$and $\leq$. on a distributive bisemilattice $(S, \cdot,+)$. The following proposition describes the relationship between those partial orders and the operation $\star$.

3.4. Proposition. If $(S, \cdot,+)$ is a distributive bisemilattice, then for all elements $x, y, z \in S$,

(i) $x \leq y$ implies $x \star z \leq y \star z$ and $z \star x \leq . z \star y$,

(ii) $x \leq_{+} y$ implies $x \star z \leq_{+} y \star z$ and $z \star x \leq_{+} z \star y$,

(iii) $x \leq_{+} x \star y \leq x$,

(iv) $x \star y \leq_{+} x \star z$ if and only if $x \star z \leq . x \star y$,

(v) $x \leq_{+} y$ implies $x \star y \leq . y \star x$,

(vi) $x \leq$. $y$ implies $x \star y \leq_{+} y \star x$,

(vii) $x \leq y \Leftrightarrow x \leq_{+} y \star x$ and $x \star y=x$,

(viii) $x \leq_{+} y \Leftrightarrow x \star y \leq . y$ and $y \star x=y$.

Proof. First, note that interchanging the operations $\cdot$ and + of $(S, \cdot,+)$ has no effect on the operation $\star$, whereas $x \leq y$ turns over to $y \leq_{+} x$ and $x \leq_{+} y$ to $y \leq x$. This shows that (i) and (ii), (v) and (vi) as well as (vii) and (viii) are equivalent, and likewise both relations contained in (iii). So it remains to show the following.

(i) Let $x \leq y$. Then $(x \star z) \cdot(y \star z)=(x \cdot y) \star(z \cdot z)=x \star z$ follows by 3.2 and $x \cdot y=x$, and shows that $x \star z \leq y \star z$. In the same way one obtains $z \star x \leq . z \star y$.

(iii) $x \leq_{+} x \star y$ follows immediately from the definition of $\star$.

(iv) Assume that $x \star y \leq_{+} x \star z$. We have to show that $x \star z=(x \star y) \cdot(x \star z)$. From 2.1 it follows that $(x \cdot y)+(x \cdot z)=(x \cdot y)+(x \cdot z)+(x \cdot y) \cdot z$, and hence

$$
\begin{aligned}
(x \star y) \cdot(x \star z) & =(x+x \cdot y) \cdot(x+x \cdot z)=x+x \cdot y+x \cdot z+x \cdot y \cdot z \\
& =x+x \cdot y+x \cdot z=x \star(y+z) \\
& =x \star y+x \star z=x \star z .
\end{aligned}
$$

The converse follows accordingly. 
(v) Assume that $x \leq_{+} y$. Then

$$
\begin{aligned}
(x \star y) \cdot(y \star x) & =(x+x \cdot y) \cdot(y+x \cdot y) \\
& =x \cdot y=x \cdot(x+y)=x \star y
\end{aligned}
$$

and hence $x \star y \leq y \star x$.

(vii) Assume that $x \leq y$. Then, using (i) we obtain $x=x \star x \leq y \star x$ and hence $x \star y=x \star(y \star x) \leq_{+}(y \star x) \star x=y \star x$ by (vi). Moreover, multiplying $x \leq y$ by $x$ from the left side and using (i) and (iii), we obtain $x=x \star x \leq x \star y \leq x$, that is, $x=x \star y$. Conversely, assume that $x=x \star y$ and $x \leq_{+} y \star x$. Then (iii) and (v) yield $x-x \star y=x \star(y \star x) \leq .(y \star x) \star x=$ $y \star x \leq y$.

The operation $\star$ is also a partition operation for $(S, \cdot,+)$ in order to obtain this bisemilattice as a Plonka sum of its maximal distributive sublattice (cf. [14], [15], [22]).

3.5. TheOREM. Let $(S, \cdot,+)$ be a bisemilattice and apply Theorem 2.2 to the corresponding left normal band $(S, \star)$ (cf. 3.1). Then $\boldsymbol{\theta}_{S}$ is also the largest congruence on $(S, \cdot,+)$ such that the equation $x \cdot y=x+y$ holds in the natural homomorphic image, which yields $i \star j=i \cdot j=i+j$ for the semilattice $(I, \star)=\left(S / \Theta_{S}, \star\right)$. Moreover, the Ptonka fibres $\left(S_{i}, \cdot,+\right)$ are the maximal distributive sublattices of $(S, \cdot,+)$ (cf. 3.3), and the Ptonka maps $p_{i, j}: S_{i} \rightarrow S_{j}$ given by $x \mapsto x \star a_{j}=x+x \cdot a_{j}$ for some $a_{j} \in S_{j}$ are also bisemilattice homomorphisms. Finally, the operations on the $\left(S_{i}, \cdot,+\right)$ and on $(I, \star)=(I, \cdot)$ determine the operations $\cdot$ and + on $S$ by

$$
a_{i}+b_{j}=a_{i} p_{i, i \cdot j}+b_{j} p_{j, i \cdot j} \text {. }
$$

The converse part of Theorem 2.2 holds accordingly.

In the sequel we call $(S, \cdot,+)$ in this context the Ptonka sum of the distributive lattices $\left(S_{i}, \cdot,+\right)$ over the semilattice $(I, \cdot)$ by the homomorphisms $\left(p_{i, j}\right)_{i, j}$ and denote the elements of $S_{i}$ by $a_{i}, b_{i}$, etc. The map $\pi: S \rightarrow I$ defined by $a_{i} \pi=i$ is called the canonical projection. This map is a bisemilattice homomorphism, too.

\section{Partially ordered left normal bands}

Let $(A, \star)$ be a semigroup of a certain type, and let $(A, \leq)$ be a partially ordered set. Then $(A, \star, \leq)$ is called a partially ordered semigroup of that type if

$$
x \leq y \text { imply } x \star z \leq y \star z \text { and } z \star x \leq z \star y
$$


for all $x, y, z \in A$. In this notion, for a left normal band $(S, \star)$ obtained form a bisemilattice $(S, \cdot,+)$ (cf. 3.1$),(S, \star, \leq)$ as well as $\left(S, \star_{,} \leq_{+}\right)$ are partially ordered left normal bands by Proposition 3.4. From the same proposition, it follows that either one of the orders $\leq$. and $\leq_{+}$determines the other, but only the former one satisfies $x \star y \leq x$. For this reason, we restrict the usual notion in this paper:

4.1. Definition. A partially ordered left normal band $(A, \star, \leq)$ is always assumed to satisfy additionally the inequality $x \star y \leq x$ for all $x, y \in A$.

There are two important examples of partially ordered left normal bands.

At first, from [21, Proposition 2.3], it follows that the relation $\leq$ defined on a left normal band $(A, \star)$ by

$$
x \leq y \Leftrightarrow x=y \star x
$$

is a partial order on $A$ (see also [6]). We claim that $(A, \star, \leq)$ is a partially ordered left normal band. Indeed, if $x \leq y$, then $x=y \star x$, and hence $(y \star z) \star(x \star z)=(y \star x) \star z=x \star z$ shows $x \star z \leq y \star z$, and $z \star x \leq z \star y$ follows similarly. Finally, $x \star y \leq x$ follows form $x \star y=x \star(x \star y)$. In particular, if $(A, \star)$ is a semilattice, then $(A, \star, \leq)$ is a commutative partially ordered left normal band.

If $(A, \star)$ is a left-zero band, that is, if $(A, \star)$ satisfies $x \star y=x$, and if $\leq$ is any partial order on $A$, then $(A, \star, \leq)$ is a partially ordered left normal band. A partially ordered left normal band of this form is called a partially ordered left-zero band.

On every partially ordered left normal band $(A, \star, \leq)$ we can define a second partial order $\subseteq$ by setting

$$
x \sqsubseteq y \Leftrightarrow x \star y \leq y \text { and } y \star x=y .
$$

The next proposition shows that the partial orders $\leq$ and $\sqsubseteq$ have analogous properties to those of $\leq$. and $\leq_{+}$in distributive bisemilattices.

4.2. Proposition. If $(A, \star, \leq)$ is a partially ordered left normal band, then the relation $\sqsubseteq$ is a partial order on $A$. Moreover, the following properties hold:

(i) $x \sqsubseteq y$ implies $x \star z \sqsubseteq y \star z$ and $z \star x \sqsubseteq z \star y$;

(ii) $x \sqsubseteq x \star y$;

(iii) $x \star y \sqsubseteq x \star z$ if and only if $x \star z \leq x \star y$;

(iv) $x \sqsubseteq y$ implies $y \star y \leq y \star x$;

(v) $x \leq y$ implies $x \star y \sqsubseteq y \star x$;

(vi) $x \leq y \Leftrightarrow x \sqsubseteq y \star x$ and $x \star y=x$;

(vii) $x \sqsubseteq y \Leftrightarrow x \star y \leq y$ and $y \star x=y$. 
Proof. A simple calculation shows that $\sqsubseteq$ is in fact a partial order on A.

(i) Suppose that $x \sqsubseteq y$. Then $x \star y \leq y$ and $y \star x=y$. Hence it follows from 4.1 that $(x \star z) \star(y \star z)=(x \star y) \star z \leq y \star z$ and $y \star z=(y \star x) \star z=$ $(y \star z) \star(x \star z)$, i.e. $x \star z \sqsubseteq y \star z$. The other inequality is shown accordingly.

(ii) We have to show that $x \star(x \star y)=(x \star y) \star x \leq(x \star y)$ and $(x \star y) \star x=$ $x \star y$. But this follows immediately from the algebraic properties of partially ordered left normal bands.

(iii) Assume that $x \star y \sqsubseteq x \star z$. Then $x \star z=(x \star z) \star(x \star y)=x \star y \star z \leq x \star y$. Conversely, if $x \star z \leq x \star y$, then $(x \star y) \star(x \star z)=x \star z \star y \leq x \star z$ and $(x \star z) \star(x \star y) \leq x \star z=(x \star z) \star(x \star z) \leq(x \star z) \star(x \star y)$, and therefore $(x \star z) \star(x \star y)=x \star z$. It follows that $x \star y \sqsubseteq x \star z$.

(iv) and (v) are trivial consequences of (vi) and (vii).

(vi) Assume that $x \leq y$. Then $x=x \star x \leq x \star y \leq x$, and hence $x=x \star y$. In addition to this equation, we have to show that $x \sqsubseteq y \star x$, that is, $x \star(y \star x) \leq y \star x$ and $(y \star x) \star x=y \star x$. The equation is again obvious, and for the inequality, note that $x \leq y$ yields $x \star(y \star x) \leq y \star(y \star x)=y \star x$. Conversely, let us assume that $x \subseteq y \star x$ and that $x \star y=x$. Then we also have $x \star(y \star x) \leq y \star x$, and therefore $x=x \star y=x \star x \star y=x \star(y \star x) \leq$ $y \star x \leq y$.

(vii) is the definition of $\sqsubseteq$.

4.3. Proposition. Let $(A, \star, \leq)$ be a partially ordered left normal band. Then for the following conditions, (i) implies (ii), and (ii) and (iii) are equivalent:

(i) the operation $\star$ is commutative;

(ii) $x \leq y$ is equivalent to $y \star x=x$;

(iii) $x \leq y$ is equivalent to $y \sqsubseteq x$.

Proof. (i) $\Rightarrow$ (ii) If $x \leq y$, then $x \leq x \star x \leq y \star x=x \star y \leq x$, and hence $x=y \star x$. Conversely, if $x=y \star x$, then $x \leq y$ by 4.1 .

(ii) $\Rightarrow$ (iii) If $x \leq y$, then by 4.2 (iii), $y \subseteq y \star x=x$. Conversely, if $y \sqsubseteq x$, then $y \star x \leq x$ and $x \star y=x$, and therefore $x \star(y \star x)=y \star x$. It follows that $x=x \star y=y \star x \leq y$.

(iii) $\Rightarrow$ (ii) Suppose that (iii) holds, and let $x \leq y$. Then, by assumption, $y \sqsubseteq x$, that is, $y \star x \leq x$ and $x \star y=x$. Hence $y \star x \leq x \star y$. By (iii), this inequality is equivalent to $x \star y \sqsubseteq y \star x$. Now by the definition of $\sqsubseteq$ this means that $x \star y=(x \star y) \star(y \star x) \leq y \star x$, and hence $x=x \star y \leq y \star x$. It follows that $x=y \star x$. Conversely, if $x=y \star x$, then $x \leq y$ by 4.1 .

The following example shows that (ii) does not imply (i). Take $A$ to be 
the free left normal band with generators $x$ and $y$. Then $A=\{x, y, x \star$ $y, y \star x\}$. Define a relation $\leq$ on $A$ by $x \star y \leq x$ and $y \star x \leq y$ and no other non-trivial comparisons.

4.4. Proposition. Let $(A, \star, \leq)$ be a partially ordered left normal band. Then the following statements are equivalent:

(i) $(A, \star, \leq)$ is a partially ordered left-zero band;

(ii) $x \star y=x$ for all $x, y \in A$;

(iii) the partial orders $\subseteq$ and $\leq$ are identical.

Proof. Clearly, if $(A, \star, \leq)$ is a partially ordered left-zero band, then $x \star y=x$ for all $x, y \in A$. If $x \star y=x$ for all $x, y \in A$, then $x \sqsubseteq y$ implies $x=x \star y \leq y \star x=y$, and, similarly, $x \leq y$ implies $x \sqsubseteq y$. Hence $\sqsubseteq=\leq$. Finally, if $\sqsubseteq=\leq$, then the rule $x \sqsubseteq x \star y \leq x$ says that $x=x \star y$.

We now would like to represent every partially ordered left normal band as a Plonka sum of partially ordered left-zero bands over a semilattice. In order to do this, we have to recall some facts.

Let $(A, \star)$ be a left normal band. It was shown in [15] that $(A, \star)$ is a Plonka sum of left-zero bands, say $\left(A_{i}, \star\right)$, over a semilattice $(I, \star)$. The decomposition of $(A, \star)$ into the Plonka sum is given by the congruence relation $\boldsymbol{\theta}_{c}$ defined as follows:

$$
\begin{aligned}
(x, y) \in \mathbf{\Theta}_{c} & \Leftrightarrow x \star y=x \text { and } y \star x=y \\
& \Leftrightarrow x, y \in A_{i} \text { for some } i \in I .
\end{aligned}
$$

The Płonka maps $p_{i, j}$ are defined by $x \mapsto x \star a_{i}$, where $a_{i} \in A_{i}$ is arbitrary. Moreover, $(I, \star)=\left(A / \Theta_{c}, \star\right)$ (see [9], [15], [22]). By Proposition 4.3 there is only one way to equip $(I, \star)$ with a partial order $\leq$ so that we obtain a partially ordered left normal band. We will use these observations in the proofs of the following results.

4.5. Proposition. Let $(A, \star, \leq)$ and $(B, \star, \leq)$ be two partially ordered left normal bands, and let $\phi: A \rightarrow B$ be $a \star$-homomorphism. Then $\phi$ preserves the partial order $\leq$ if and only if $\phi$ preserves the partial order $\sqsubseteq$. Moreover, if $(B, \star)$ is commutative, then every $\star$-homomorphism $\phi$ preserves both partial orders.

Proof. The first statement follows immediately from 4.2. Now assume that $(A, \star)$ is a Plonka sum of left-zero bands $\left(A_{i}, \star\right)$ over a semilattice $(I, \star)$ and that $(B, \star)$ is commutative. It follows form 4.3 that in $(B, \star, \leq)$ we have $u \leq v$ if and only if $v \star u=u$. Now let $x \leq y$ in $(A, \leq)$. We have to show that $x \phi \leq y \phi$, that is, $x \phi=(y \star x) \phi$. First, note that $x \star(y \star x)=x$ : Indeed, $x \leq y$ implies $x \leq x \star x \star x \leq x \star y \star x \leq x$, 
and hence $x=x \star(y \star x)$. Using the commutativity of $B$, we obtain $x \phi=(x \star(y \star x)) \phi=x \phi \star(y \star x) \phi=(y \star x) \phi \star x \phi=(y \star x \star x) \phi=(y \star x) \phi$.

Now assume that $(A, \star, \leq)$ is a partially ordered left normal band, and let $\pi: A \rightarrow A / \Theta_{c}=I$ be the canonical quotient map. It follows that, in the partial order and algebraic structure induced from $(A, \star, \leq)$, the preimage $A_{i}=i \pi^{-1}$ is also a partially ordered left-zero normal band for each $i \in I$. Moreover, if $i \leq j$ in $(I \star)$, then the Plonka map $p_{j, k}: A_{j} \rightarrow A_{i}$ is given by $x \mapsto x \star a_{i}$, and therefore preserves the partial orders.

4.6. Proposition. Let $(A, \star, \leq)$ be a partially ordered left normal band, and let $(I, \star), A_{i}$ and $A_{j}$ be defined as above. If $x \in A_{i}$ and $y \in A_{j}$, then

$$
x \leq y \Leftrightarrow i \leq j \text { and } x \leq y \star a_{i}=y p_{j, i} .
$$

Proof. Assume that $x \leq y$. Then, since the mapping $\pi: A \rightarrow A / \Theta_{c}=I$ preserves the partial orders, we obtain $i=x \pi \leq y \pi=j$. Moreover, since $x \in A_{i}, x \star a_{i}=x$ and therefore $x=x \star a_{i} \leq y \star a_{i}$. Conversely, if $x \leq y \star a_{i}$, then $x \leq y \star a_{i} \leq y$, that is, $x \leq y$.

Conversely, let $(I, \star, \leq)$ be a partially ordered commutative left normal band (that is, let $(I, \star)$ be a semilattice, and the partial order to be defined by $i \leq j$ if and only if $i \star j=i)$. For each $i \in I$, let $\left(A_{i}, \star, \leq\right)$ be a partially ordered left-zero band. If $i \leq j$, then let $p_{j, i}: A_{j} \rightarrow A_{i}$ be a band homomorphism that preserves $\leq$. Suppose that the family of the $p_{j, i}$ 's satisfies the conditions for a Plonka sum. Then the Plonka sum $(A, \star)$ of the $\left(A_{i}, \star\right)$ is a left normal band (see [9], [15], [22]). Define a partial order $\leq$ on $A$ by

if $x \in A_{i}$ and $y \in A_{j}$ then $x \leq y \Leftrightarrow i \leq j$ and $x \leq y p_{j, i}$.

4.7. Proposition. Let $(A, \star, \leq)$ be constructed as above. Then $(A, \star, \leq)$ is a partially ordered left normal band.

Proof. It is a routine computation to show that the relation $\leq$ is a partial order. We have to verify the conditions of 4.1. In the following proof, if $i, j \in I$ are given, we will abbreviate $i j=i \star j$. To show that (i) holds, let $x \leq y$ for $x \in A_{i}$ and $y \in A_{j}$. Then $i \leq j$ and $x \leq y p_{j, k}$. Let $z \in A_{k}$. Clearly $i k \leq j k$. We want to show that $x \star z \leq(y \star z) p_{j k, i k}$. Indeed,

$$
\begin{aligned}
x \star z & =\left(x p_{i, i k}\right) \star z p_{k, i k}=x p_{i, i k} \leq\left(y p_{j, i}\right) p_{i, i k} \quad\left(\text { since } x \leq y p_{j, i}\right) \\
& =y p_{j, i k}=y p_{j, i k} \star z p_{k, i k}=\left(y p_{j, j k} \star z p_{k, j k}\right) p_{j k, i k}=(y \star z) p_{j k, i k} .
\end{aligned}
$$

The conditions (ii) and (iii) can be checked similarly.

We call the partially ordered left normal band $(A, \star, \leq)$ the partially ordered Plonka sum of the $\left(A_{i} \star, \leq\right)$ over $(I, \star, \leq)$. 
4.8. Theorem. Every partial ordered left normal band is isomorphic to the partially ordered Plonka sum of partially ordered left-zero bands.

Let $(3, \cdot,+)$ be the subdirectly irreducible 3 -element distributive bisemilattice $\mathbf{3}=\{0,1, \alpha\}$, where the operations + and $\cdot$ are given as follows:

$$
\begin{array}{cc}
1+0=1, & 0 \cdot 1=0, \\
0+\alpha=1+\alpha=\alpha, & 0 \cdot \alpha=1 \cdot \alpha=\alpha .
\end{array}
$$

Then we have

$$
\alpha \leq 0 \leq .1 \text { and } 0 \leq_{+} 1 \leq_{+} \alpha .
$$

On 3 , the operation $\star$ is given by

$$
x \star y= \begin{cases}x & \text { for } y \neq \alpha \\ y & \text { for } y=\alpha .\end{cases}
$$

It follows that $(3, \star, \leq)$ is partially ordered left normal band. It is easy to see that on 3 the relations $\leq_{+}$and $\sqsubseteq$ coincide.

We would like to show that for every partially ordered left normal band $(A, \star, \leq)$, the family of all partial order preserving band homomorphisms $\phi: A \rightarrow 3$ separates the points of $A$. We are going to need the following lemma.

4.9. Lemma. Let $(A, \star, \leq)$ be the partially ordered Plonka sum of partially ordered left-zero bands $\left(A_{i}, \star, \leq\right)$ over a semilattice $(I, \star) ;$ let $p_{j, i}: A_{j}$ $\rightarrow A_{i}$ be Plonka homomorphisms, and let $\pi: A \rightarrow I$ be the canonical projection. Moreover, for $i \in I$ let $\phi_{i}: A_{i} \rightarrow\{0,1\} \subset 3$ be order preserving. One easily checks that $\phi_{i}$ is a homomorphism of $\left(A_{i}, \star\right)$ into $(3, \star)$. Then the mapping

$$
\phi^{i}: A \rightarrow 3, x \mapsto \begin{cases}\left(x p_{x \pi, i}\right) \phi & \text { for } i \leq x \pi \\ \alpha & \text { otherwise }\end{cases}
$$

is an order preserving band homomorphism extending $\phi_{i}$.

Proof. We first show that $\phi^{i}$ is a band homomorphism. Let $x, y \in A$, and let $j=x \pi$ and $k=y \pi$. If $i \leq j, k$, then $i \leq j \star k=(x \star y) \pi$, and hence, using the definition of Ptonka sums, we obtain

$$
\begin{aligned}
(x \star y) \phi^{i} & =(x \star y) p_{(x \star y) \pi, i} \phi_{i}=\left(x p_{j,(x \star y) \pi} \star y p_{k,(x \star y) \pi}\right) p_{(x \star y) \pi, i} \phi_{i} \\
& =\left(x p_{j,(x \star y) \pi} p_{(x \star y) \pi, i} \star y p_{k,(x \star y) \pi} p_{(x \star y) \pi, i}\right) \phi_{i} \\
& =\left(x p_{j, i} \star y p_{k, i}\right) \phi_{i}=\left(x p_{j, i} \phi_{i}\right) \star\left(y p_{k, i} \phi_{i}\right)=\left(x \phi^{i}\right) \star\left(y \phi^{i}\right) .
\end{aligned}
$$


If either $i \not j$ or $i \not k$, then $i \not(x \star y) \pi$, and therefore $(x \star y) \phi^{i}=\alpha$ and either $z \phi^{i}=\alpha$ or $y \phi^{i}=\alpha$. Therefore $\left(x \phi^{i}\right) \star\left(y \phi^{i}\right)=\alpha=(x \star y) \phi^{i}$. Hence $\phi^{i}$ is a band homomorphism.

It remains to show that $\phi^{i}$ is order preserving, that is, $x \leq y$ in $A$ implies $x \phi^{i} \leq y \phi^{i}$ in 3. If $j=x \pi$ and $k=y \pi$, then $x \leq y$ implies $j \leq k$ and $x \leq y p_{k, j}$. Hence if $i\left\lfloor j\right.$, then $x \phi^{i}=\alpha \leq y \phi^{i}$. Now assume that $i \leq j \leq k$. In this case, $x \phi^{i}=\left(x p_{j, k}\right) \phi_{i} \leq\left(y p_{k, j} p_{j, k}\right) \phi_{i}=y p_{k, i} \phi_{i}=y \phi^{i}$.

4.10. Proposition. Let $(A, \star, \leq)$ be a partially ordered left normal band, and let $x, y \in A$. If $x \$ y$, then there is an order preserving band homomorphism $\phi: A \rightarrow 3$ such that $x \phi \Varangle . y \phi$. Similarly, if $x \notin y$, then there is an order preserving band homomorphism $\phi: A \rightarrow 3$ such that $x \phi \Varangle_{+} y \phi$.

Proof. Let $I=A / \Phi_{c}$ be the largest semilattice quotient of $A$, and let $\pi: A \rightarrow I$ be the canonical quotient map. For each $i \in I$, let $A_{i}=i \pi^{-1}$, and let $p_{j, i}: A_{j} \rightarrow A_{i}, i \leq j$, be the Ptonka maps. Now assume that $x, y \in A$ and that $x \$ y$. Let $i=x \pi$ and $j=y \pi$. By our previous discussion, there are two possibilities.

CASE I. $x \pi \ddagger y \pi$. In this case, let $\phi_{i}: A_{i} \rightarrow\{0,1\}$ be any order preserving map, and let $\phi^{i}: A \rightarrow 3$ be the extension of $\phi_{i}$ to $A$ as constructed in 4.9. Then $x \phi^{i} \in\{0,1\}$ and $y \phi^{i}=\alpha$, and hence $x \phi^{i} \not . y \phi^{i}$.

CASE II. $x \pi \leq y \pi$ and $x \not y p_{j, i}$. In this case, let $\phi_{i}: A_{i} \rightarrow\{0,1\}$ be an order preserving map such that $x \phi_{i}=1$ and $\left(y p_{j, i}\right) \phi_{i}=0$. Then $\phi^{i}$ maps $x$ to 1 and $y$ to 0 , hence $x \phi^{i} \not$. $y \phi^{i}$.

For the proof of the second statement, recall that on 3 , the relations $\leq_{+}$ and $\subseteq$ are identical. Hence this assertion follows immediately from the definition of $\sqsubseteq$.

4.11. THEOREM. Every partially ordered left normal band is isomorphic to a partially ordered subalgebra of a power of 3 .

Proof. Let $X$ be the set of all order preserving homomorphisms $\phi: A \rightarrow$ 3. Let $\varepsilon: A \rightarrow 3^{X}$ be defined by evaluation, that is, $(\phi)(x \varepsilon)=x \phi$. Then it follows immediately from 4.10 that $\varepsilon$ is an embedding.

We also need the idea of left normal bands with constants. If $A$ is of the form $3^{x}$ for a certain set $X$, then the constants correspond to the constant maps. Hence we make the following definition:

4.12. Definition. A partially ordered left normal band with constants is a partially ordered left normal band $(A, \star, \leq)$ together with three constants 
$c_{0}, c_{1}, c_{\alpha}$ such that the following properties are satisfied:

$$
\begin{gathered}
x \star c_{\alpha}=c_{\alpha} \star x=c_{\alpha}, \quad x \star c_{0}=x \star c_{1}=x, \\
c_{0} \sqsubseteq x \leq c_{1}, \quad c_{\alpha} \leq x \sqsubseteq c_{\alpha}, \\
c_{0} \star x=c_{1} \star x \Rightarrow x=c_{\alpha} .
\end{gathered}
$$

If $\phi: A \rightarrow 3$ is an order preserving band homomorphism such that $c_{0} \phi=0$, $c_{1} \phi=1$, and $c_{\alpha} \phi=\alpha$, then we say that $\phi$ preserves the constants.

4.13. Proposition. Let $\left(A, \star, \leq, c_{0}, c_{1}, c_{\alpha}\right)$ be a partially ordered left normal band with constants, and assume that $(A, \star, \leq)$ is the partially ordered Ptonka sum of $\left(A_{i}, \star, \leq\right)$ over its semilattice replica $(I, \star)$. Then $(I, \star)$ has a largest and a smallest element and each $\left(A_{i}, \leq\right)$ has a largest element.

Proof. First, note that $c_{1} \pi$ and $c_{\alpha} \pi$ are the largest and the smallest element of $(I, \star)$. Now by Definition 4.12 , both constants $c_{0}$ and $c_{1}$ are in the same Plonka fibre $A_{1}=A_{c_{1} \pi}$. By the same definition and Proposition 4.4, it follows, for each element $x_{1} \in A_{1}$, that $c_{0} \leq x_{1} \leq c_{1}$. Since for each element $x_{i} \in A_{i}, x_{i} \leq c_{1}$ and for arbitrary $y_{i} \in A_{i}, c_{1} \star x_{i}=c_{1} \star y_{i}$, it follows by Proposition 4.6 that $x_{i} \leq c_{1} p_{1, i}=c_{1} \star x_{i}$. Therefore $c_{1} \star x_{i}$ is the largest element of $\left(A_{i}, \leq\right)$.

Note that the constants form a bichain

$$
c_{\alpha} \leq c_{0} \leq c_{1}, \quad c_{0} \sqsubseteq c_{1} \sqsubseteq c_{\alpha} .
$$

Moreover, $A_{c_{\alpha} \pi}=\left\{c_{\alpha}\right\}$ and if for some $i \in I, c_{0} p_{1, i}=c_{1} p_{1, i}$, then $i=c_{\alpha} \pi$ and $A_{i}=\left\{c_{\alpha}\right\}^{\pi}$.

Clearly, $\left(\mathbf{3}, \star, \leq ., c_{0}, c_{1}, c_{\alpha}\right)$ is a partially ordered left normal band with constants $c_{0}=0, c_{1}=1$, and $c_{\alpha}=\alpha$, and therefore every subalgebra of $\left(3^{X}, \star, \leq\right)$ that contains the constant maps with values 0,1 , and $\alpha$ is a partially ordered left normal band with constants. We would like to show that every partially ordered let normal band with constants is of this form.

4.14. Proposition. Let $\left(A, \star, \leq, c_{0}, c_{1}, c_{\alpha}\right)$ be an partially ordered left normal band with constants, and let $\phi: A \rightarrow \mathbf{3}$ be an order preserving band homomorphism. If $\phi$ is surjective, then $\phi$ preserves the constants.

Proof. Since $c_{\alpha}$ is minimal with respect to $\leq$, the image of $c_{\alpha}$ has to be minimal with respect to $\leq$, in 3 , and it follows that $c_{\alpha} \phi=\alpha$. Similarly, since $c_{1}$ is maximal with respect to $\leq$, it follows that $c_{1} \phi=1$, and since $c_{0}$ is minimal with respect to $\sqsubseteq$, it follows that $c_{0} \phi=0$. 
4.15. Proposition. Let $\left(A, \star, \leq, c_{0}, c_{1}, c_{\alpha}\right)$ be a partially ordered left normal band with constants, and let $x, y \in A$. If $x \notin y$, then there is an order preserving band homomorphism $\phi: A \rightarrow 3$ that preserves the constants such that $x \phi \Varangle . y \phi$. Similarly, if $x \notin y$, then there is an order preserving band homomorphism $\phi: A \rightarrow 3$ that preserves the constants such that $x \phi \Varangle_{+} y \phi$.

Proof. We use the same notations as in the proof of 4.10. Again we have two cases to consider.

CASE I. assume that $x \pi \not y \pi$. Obviously, $c_{1} \star x, c_{0} \star x \in A_{i}$, and since $x \neq c_{\alpha}$, it follows that $c_{1} \star x \neq c_{0} \star x$. Hence $\left|A_{i}\right| \geq 2$, and we can find an order preserving map $\phi_{i}: A_{i} \rightarrow\{0,1\}$ such that $A_{i} \phi_{i}=\{0,1\}$. The map $\phi^{i}: A \rightarrow 3$ is then surjective, hence preserves the constants, and satisfies $x \phi_{.}^{\ddagger} y \phi^{i}=\alpha$.

CASE II. $x \pi \leq y \pi$ and $x \not y p_{j, i}$. This case is analogous to Case II in the proof of 4.10 . Note that the map constructed there is surjective, and therefore preserves the constants by 4.14 .

The following theorem is an easy consequence of 4.15 .

4.16. THEOREM. Every partially ordered left normal band with constants $\left(A, \star, \leq, c_{0}, c_{1}, c_{\alpha}\right)$ is isomorphic to a partially ordered subalgebra of $a$ power of $\left(3, \star, \leq, c_{0}, c_{1}, c \alpha\right)$ containing the constants.

Proof. Let $X$ be the set of all order preserving homomorphisms $\phi: A \rightarrow 3$ that preserve the constants, and let $\varepsilon: A \rightarrow 3^{X}$ be defined by evaluation, that is, $(\phi)(x \varepsilon)=x \phi$. Then it follows immediately from 4.15 that $\varepsilon$ is an embedding of left normal partially ordered bands with constants.

We conclude this section with an important example.

Let $(S, \cdot,+)$ and $(T, \cdot,+)$ be two distributive bisemilattices, and let $A=\operatorname{Hom}_{b}(S, T)$, the set of all bisemilattice homomorphisms from $S$ into $T$. Then, $A$ is a subset of the bisemilattice $\left(T^{S}, \cdot,+\right)$, where the operations - and + are defined pointwise. If we view $T^{S}$ as a partially ordered left normal band in the operation $f \star g=f+f \cdot g$, then $A$ is even closed under $\star$. The proof of these statements follows easily from the entropic laws 3.2. Moreover, the constant maps with values 0,1 , and $\alpha$, respectively, belong to $\operatorname{Hom}_{b}(S, 3)$. hence $\operatorname{Hom}_{b}(S, 3)$ is a subalgebra of $\left(3^{S}, \star, \leq, c-0, c_{1}, c_{\alpha}\right)$, and therefore

4.17. THEOREM. If $(S, \cdot,+)$ and $(T, \cdot,+)$ are two distributive bisemilattices, then $\left(\operatorname{Hom}_{b}(S, T), \star, \leq, c_{0}, c_{1}, c_{\alpha}\right)$ is a partially ordered left normal band with constants. 
Problem. Assume that $(S, \cdot,+)$ and $(T, \cdot,+)$ are two distributive bisemilattices. Is there a relation between the Ptonka sums for $S$ and $T$ and the Plonka sum for $\operatorname{Hom}_{b}(S, T)$ ? We will find lots of hints for such a relation in the case where $T=3$. Actually, it is possible to show that the Plonka sum for $\operatorname{Hom}_{b}(S, 3)$ is the "dual" of the Plonka sum for $S$.

\section{Order preserving mappings from bi-ordered set into 3}

A set $A$ equipped with two partial orders $\leq$ and $\sqsubseteq$ is called a bi-ordered set and is denoted by $(A, \leq, \sqsubseteq)$.

For a bi-ordered set $(A, \leq, \subseteq)$, define $\operatorname{Ord}(A)$ to be the set of all mappings $\phi:(A, \leq, \sqsubseteq) \rightarrow\left(3, \leq, \leq_{+}\right)$that preserve both partial orders, that is, if $a, b \in A$, and if $a \leq b$, then $a \phi \leq b \phi$. and if $a \sqsubseteq b$, then $a \phi \leq_{+} b \phi$.

In order to describe the structure of $\operatorname{Ord}(A)$, we shall need the following

5.1. Lemma. Let $x, y, u, v \in 3$. Then the inequality $x \cdot u+y \cdot v \leq_{+}$ $x \cdot y+u \cdot v$ does not hold if and only if $x=u=0$ and $y=v=1$ or if $x=u=1$ and $y=v=0$.

Proof. Assume that $\alpha \in\{x, y, u, v\}$, say $x=\alpha$. Then

$$
\begin{aligned}
x \cdot u+y \cdot v & =\alpha u+y \cdot v=\alpha+y \cdot v=\alpha \\
& =\alpha+u \cdot v=\alpha y+u \cdot v=x \cdot y+u \cdot v .
\end{aligned}
$$

Hence, if the above inequality were not true, then $x, y, u, v \in\{0,1\}$. Assume that $x \neq u$, say $x=0$ and $u=1$. Then, since $\leq_{+}$and $\leq$agree on the set $\{0,1\}$, we obtain

$$
x \cdot u+y \cdot v=y \cdot v \leq_{+} v=0+v \cdot 1=x \cdot y+u \cdot v .
$$

Hence, if this inequality does not hold, it follows that $x=u$ and similarly $y=v$. Clearly, $x=u=y=v$ is impossible, and therefore either $x=u=0$ and $y=v=1$ or $x=u=1$ and $y=v=0$.

Conversely, if either $x=u=0$ and $y=v=1$ or $x=u=1$ and $y=v=0$, then $x \cdot u+y \cdot v=1$ and $x \cdot y+u \cdot v=0$, and therefore the inequality $x \cdot u+y \cdot v \leq_{+} x \cdot y+u \cdot v$ is incorrect.

5.2. Proposition. Let $(A, \leq, \sqsubseteq)$ be a bi-ordered set. The $\operatorname{Ord}(A)$ is a subbisemilattice of $\left(3^{A}, \cdot,+\right)$, where the operations in $3^{A}$ are defined pointwise. 
Proof. Let $\phi, \psi \in \operatorname{Ord}(A)$. We have to show that $\phi+\psi, \phi \cdot \psi \in \operatorname{Ord}(A)$, where

$$
a(\phi+\psi)=a \phi+a \psi \text { and } a(\phi \cdot \psi)=a \phi \cdot a \psi .
$$

More explicitly, we have to verify the following.

(i) If $a, b \in A$ and if $a \leq b$, then $a(\phi \cdot \psi) \leq b(\phi \cdot \psi)$ and $a(\phi+\psi) \leq$ $b(\phi+\psi)$.

(ii) If $a, b \in A$ and if $a \sqsubseteq b$, then $a(\phi \cdot \psi) \leq_{+} b(\phi \cdot \psi)$ and $a(\phi+\psi) \leq_{+}$ $b(\phi+\psi)$.

We will show (i) only; (ii) follows similarly. In order to verify (i), pick $a, b \in A$ and assume that $a \leq b$. Then

$$
a \phi \leq . b \phi, \quad a \psi \leq . b \psi,
$$

that is,

$$
a \phi \cdot b \phi=a \phi, \quad a \psi \cdot b \psi=a \psi
$$

It follows that

$$
a(\phi \cdot \psi) \cdot b(\phi \cdot \psi)=a \phi \cdot b \phi \cdot a \psi \cdot b \psi=a \phi \cdot a \psi=a(\phi \cdot \psi)
$$

and hence

$$
a(\phi \cdot \psi) \leq . b(\phi \cdot \psi) .
$$

Moreover, since 3 is a distributive bisemilattice, we have

$$
\begin{aligned}
a(\phi+\psi) \cdot b(\phi+\psi) & =a \phi \cdot b \phi+a \phi \cdot b \psi+a \psi \cdot b \phi+a \psi \cdot b \psi \\
& =a \phi+a \psi+(a \phi \cdot b \psi+a \psi \cdot b \phi) .
\end{aligned}
$$

Thus, we have to show that $a \phi \cdot b \psi+a \psi \cdot b \phi \leq_{+} a \phi+a \psi=a \phi \cdot b \phi+b \psi \cdot a \psi$. By Lemma 5.1 this inequality can only be violated if either $a \phi=b \psi=0$ and $b \phi=a \psi=1$ or $a \phi=b \psi=1$ and $b \phi=a \psi=0$. The first possibility represents a contradiction to $a \psi \leq b \psi$, and the second case conflicts with $a \phi \leq, b \phi$. It follows that $a(\phi+\psi) \leq . b(\phi+\psi)$.

Problem. As a distributive bisemilattice, $(\operatorname{Ord}(A), \cdot,+)$ is a Plonka sum of distributive lattices $\left(S_{i}, \cdot,+\right)$ over a semilattice $(I, \cdot)$. Is it possible to discover $(I, \cdot)$ and the $\left(S_{i}, \cdot,+\right)$ 's inside of $(\operatorname{Ord}(A), \cdot,+)$ in terms of $(A, \cdot,+)$ and its decomposition as a Ptonka sum?

Now let $(A, \star, \leq)$ be a partially ordered left normal band. If $\phi, \psi: A \rightarrow 3$ are two band homomorphisms, then the entropic laws 3.2 imply that $\phi+\psi$ and $\phi \cdot \psi$ are again band homomorphisms. Hence, if $\operatorname{Hom}_{\star}(A, 3)$ denotes the collection of all band homomorphisms from $A$ into 3 , then $\operatorname{Hom}_{\star}(A, 3)$ is a sub-bisemilattice of $\left(3^{A}, \cdot,+\right)$, and therefore a distributive bisemilattice. Define

$$
\tilde{A}=\operatorname{Ord}(A) \cap \operatorname{Hom}_{\star}(A, 3) \text {. }
$$


5.3. Proposition. If $(A, \star, \leq)$ is a partially ordered left normal band, then $(\tilde{A}, \cdot,+)$ is a distributive bisemilattice.

If $A$ is a partially ordered left normal band with constants, let

$$
\widetilde{A}^{c}=\{\phi \in \widetilde{A}: \phi \text { preserves the constants }\} \text {. }
$$

5.4. Proposition. If $\left(A, \star, \leq c_{0}, c_{1}, c_{\alpha}\right)$ is a partially ordered left normal band with constants, then $\left(\widetilde{A}^{c}, \cdot,+\right)$ is a distributive bisemilattice.

\section{Compact totally disconnected partially ordered left normal bands}

In this section, we finally define the dual category to the category of all distributive bisemilattices.

6.1. Definition. A compact totally disconnected partially ordered left normal band is partially ordered left normal band with constants $(A$, *, $\leq, c_{0}, c_{1}, c_{\alpha}$ ) together with a compact totally disconnect topology $\tau$ such that $(A, \leq, \tau)$ is totally order disconnected, and such that the mapping $\star: A \times A \rightarrow A$ is continuous with respect to $\tau$.

(Recall that a partially ordered topological space $(X, \leq, \tau)$ is called totally order disconnected if (a) $\{(x, y) \in X \times X: x \leq y\}$ is closed, and (b) if $x, y \in X$, and if $x \notin y$, then there is an open and closed lower set $U$ such that $y \in U$ and $x \notin U$.)

It follows easily from the definition of $\subseteq$ that for every compact totally disconnected partially ordered left normal band the partially ordered space $(A, \sqsubseteq, \tau)$ is also totally order disconnected. A proof of this statement follows also from 6.5 , and we will not use this fact in the remainder of this section.

Typical examples of such objects are obtained as follows: let us consider 3 as a partially ordered left normal band with constants. Equip 3 with the discrete topology, and for every set $X$, equip $3^{X}$ with the product topology. Then 3 is a compact totally disconnected partially ordered left normal band with constants, and so is every closed subalgebra of $\left(3^{X}, \star\right)$ that contains the constants. In order to show that every compact totally disconnected partially ordered left normal band is of this form, we need topological versions of 4.15 and 4.16 .

In a first step, we show that the semilattice replica of a compact totally disconnected left normal band is algebraic.

6.2. Proposition. Let $\left(A, \star, \leq, c_{0}, c_{1}, c_{\alpha}, \tau\right)$ be a compact totally disconnected partially ordered left normal band with constants. 
(i) The image of the map $A \rightarrow A, x \mapsto a \star x$ is a closed subsemilattice of $(A, \star)$ with a largest element, and hence is an algebraic lattice.

(ii) The semilattice replica of $(A, \star)$ is an algebraic lattice.

Proof. (i) Since $(a \star x) \star(a \star y)=a \star x \star y=a \star y \star x=(a \star y) \star(a \star x)$, it follows that the image of $x \mapsto a \star x$ is a commutative band, and hence a semilattice. Clearly, the image is compact, and hence closed. Therefore, the image of $x \mapsto a \star x$ is a compact totally disconnected semilattice with $a=a \star c_{1}$ as largest element, and every such semilattice is in fact an algebraic lattice.

(ii) For each $c \in A$, let $A_{c}=\{c \star x: x \in A\}$. Define a map $A \rightarrow \prod_{c \in A} A_{c}$ by $x \mapsto(c \star x)_{c \in A}$. The image of this map is a compact subsemilattice of the algebraic lattice $\prod_{c \in A} A_{c}$ containing a largest element, and hence it is an algebraic lattice. Now note that the kernel of this map consists of all pairs $(x, y)$ such that $c \star x=c \star y$ for all $c \in A$. Since in a left normal band we have

$$
(\forall c \in A c \star x=x \star y) \Leftrightarrow(x \star y=x \text { and } y \star x=y)
$$

we conclude that $\Phi_{c}$ is the kernel of the map $x \mapsto(c \star x)_{c \in A}$, and therefore the image of this map is the semilattice replica of $(A, \star)$.

There is an alternative proof of 6.2. It can be shown that the semilattice replica of $(A, \star)$ is actually isomorphic with the image of the map $x \mapsto c_{1} \star x$, and this approach would even give a more concrete representation of the semilattice replica.

Now let $\left(A, \star, \leq, c_{0}, c_{1}, c_{\alpha}, \tau\right)$ be a compact totally disconnected left normal band with constants. Let $(I, \star)$ be the semilattice replica of $(A, \star)$, and represent $(A, \star)$ as a Plonka sum of $\left(A_{i}, \star\right), i \in I$. Recall that the maps $p_{j, i}: A_{j} \rightarrow A_{i}$ are given by $x \mapsto x \star a_{i}$ where $a_{i} \in A_{i}$ is arbitrary. hence all the maps $p_{j, i}: A_{j} \rightarrow A_{i}$ are continuous. If $\phi_{i}: A_{i} \rightarrow\{0,1\} \subset \mathbf{3}$ is an order preserving mapping, then we extended this map to a map

$$
\phi^{i}: A \rightarrow 3, x \mapsto \begin{cases}\left(x \star a_{i}\right) \phi_{i} & \text { if } x \in A_{j}, i \leq j \\ \alpha & \text { otherwise. }\end{cases}
$$

In Section 3 we saw already that this map $\phi^{i}$ is an order preserving band homomorphism. Moreover, $\phi^{i}$ preserves the constants if and only if $\phi_{i}$ maps $A_{i}$ onto $\{0,1\}$ and if $i$ is not the smallest element of $(I, \star)$.

6.3. Proposition. $\phi^{i}$ is continuous if and only if $i \in I$ is a compact element and $\phi_{i}: A_{i} \rightarrow\{0,1\}$ is continuous.

Proof. First, assume that $\phi^{i}$ is continuous. Then $F=\left\{x \in A: x \phi^{i}=\alpha\right\}$ and $G=\left\{x \in A: x \phi^{i} \neq \alpha\right\}$ are two open and closed sets. Since the canonical 
map $\pi: A \rightarrow I$ is continuous, and since $F$ and $G$ are unions of congruence classes of $\Theta_{c}$, it follows that both $F=F \pi \pi^{-1}$ and $G=G \pi \pi^{-1}$ are both open and closed. Since $\pi: A \rightarrow I$ is continuous and since $A$ and $I$ are compact spaces, it is a (topological) quotient map. Therefore $F \pi$ and $G \pi$ are open and closed. But $G \pi=\{j: i \leq . j\}$. Hence $i$ is a compact element (see [8]). Moreover, since the restriction of $\phi^{i}$ to $A_{i}$ is equal to $\phi_{i}$, we conclude that $\phi_{i}$ is continuous.

Conversely, assume that $i \in I$ is compact and that $\phi_{i}: A_{i} \rightarrow\{0,1\}$ is continuous. Then the map

$$
G=\bigcup_{i \leq j} A_{j} \rightarrow\{0,1\}, \quad x \mapsto\left(x \star a_{i}\right) \phi_{i},
$$

is continuous (as the composition of the continuous maps $\phi_{i}$ and $x \mapsto x \star a_{i}$ ), and the set $\bigcup_{i \leq . j} A_{j}$ is open and closed (as the preimage of $\{j: i \leq . j\}$ under the continuous map $\pi: A \rightarrow I$ ). Since $\phi^{i}$ has the constant value $\alpha$ on the complement of $\bigcup_{i \leq j} A_{j}$, it is also continuous there, and it follows that $\phi^{i}$ is continuous.

6.4. Proposition. Let $\left(A, \star, \leq, c_{0}, c_{1}, c_{\alpha}, \tau\right)$ be a compact totally disconnected partially ordered left-normal band with constants, and let $x, y \in A$. If $x \$ y$, then there is a continuous order preserving band homomorphism $\phi: A \rightarrow 3$ that preserves the constants such that $x \phi \$ . y \phi$. Similarly, if $x \notin y$, then there is a continuous order preserving band homomorphism $\phi: A \rightarrow 3$ that preserves the constants such that $x \phi \Varangle_{+} y \phi$.

Proof. This proof is very similar to the proof of 4.15. Again, we represent $(A, \star)$ as the Plonka sum of $\left(A_{i}, \star\right), i \in I$. let $\pi: A \rightarrow I$ be the canonical map. For each $i \in I$ pick an element $a_{i} \in A_{i}$. Let $i_{x}=x \pi$ and let $i_{y}=y \pi$. There are two cases to consider.

CASE I. Assume that $i_{x}=x \pi \nsubseteq y \pi=i_{y}$. Since $I$ is an algebraic lattice, there is a compact element $i_{0} \leq i_{x}$ such that $i_{0} \npreceq i_{y}$. Moreover, we may assume that $i_{0}$ is not the smallest element $c_{\alpha} \pi$ of $I$. Hence it follows that $A_{i_{0}}$ contains at least two distinct elements, namely $c_{0} \star a_{i_{0}}$ and $c_{1} \star a_{i_{0}}$. Since $A_{i_{0}}$ is a closed subspace of the order disconnected space $A$, it follows that $A_{i_{0}}$ is totally order disconnected itself. Hence by the definition of totally order disconnectedness, there is a continuous order preserving surjective map $\phi_{i_{0}}: A_{i_{0}} \rightarrow\{0,1\}$. The map $\phi^{i_{0}}$ maps $y$ to $\alpha$ and satisfies $x \phi^{i_{0}} \in\{0,1\}$. Hence $x \phi^{i_{0}} \ddagger . y \phi^{i_{0}}$. Since $\phi^{i_{0}}$ is surjective, it preserves the constants. Hence the map $\phi=\phi^{i_{0}}$ has the required properties. 
CASE II. Assume that $i_{x}=x \pi \leq y \pi=i_{y}$. Then, since $x \notin y$, it follows that $x \notin y \star a_{i_{x}}$. For every compact element $i \leq i_{x}$ let $y_{k}=y \star a_{k}=$ $y \star a_{i_{x}} \star a_{k}$ and $x_{k}=x \star a_{k}$, where the first equality follows from $a_{i_{x}} \star a_{k} \in A_{k}$ and the fact that the value of $y \star a_{k}$ does not depend on the particular choice of $a_{k} \in A_{k}$. Now assume that for each compact $k \leq i_{x}$ we had $x_{k} \leq y_{k}$. Pick a convergent subnet of the net $\left(a_{k}\right)_{k}$, where $k$ ranges over all compact element below $i_{x}$. Let $a$ be the limit point of $\left(a_{k}\right)_{k}$. Since $\pi$ is continuous, and since the net of all compact elements below $i_{x}$ converges to $i_{x}$, it follows that $a \in A_{i_{x}}$, and therefore $x \star a=x$ and $y \star a=y \star a_{i_{x}}$. Now $x_{k} \leq y_{k}$ for all $k$ would imply

$$
\begin{aligned}
x & =x \star a=x \star \lim _{k} a_{k}=\lim _{k} x \star a_{k}=\lim _{k} x_{k} \\
& \leq \lim _{k} y_{k}=\lim _{k} y \star a_{k}=y \star \lim _{k} a_{k}=y \star a=y \star a_{i_{x}},
\end{aligned}
$$

a contradiction. Hence we can find a compact element $k \leq i_{x}$ such that $x \star a_{k} \nless y \star a_{k}$. Now let $\phi_{k}: A_{k} \rightarrow\{0,1\}$ be any continuous order preserving map so that $\left(x \star a_{k}\right) \phi_{k}=1$ and $\left(y \star a_{k}\right) \phi_{k}=0$. The map $\phi^{k}: A \rightarrow 3$ then is surjective, and satisfies $y \phi^{k}=0, x \phi^{k}=1$, hence has all the required properties.

6.5. Theorem. Let $\left(A, \star, \leq, c_{0}, c_{1}, c_{\alpha}, \tau\right)$ be a compact totally disconnected partially ordered left normal band with constants. Then $(A, \star$, $\left.\leq, c_{0}, c_{1}, c_{\alpha}, \tau\right)$ is algebraically and topologically isomorphic to a closed subalgebra of $\left(3^{X}, \star, \leq, c_{0}, c_{1}, c_{\alpha}, \tau^{\prime}\right)$ for a certain index set $X$.

Proof. The proof of this theorem is similar to the proof of 4.16 , but we use 6.4 instead of 4.15 .

\section{The duality}

Let $\mathscr{D} \mathscr{B}$ be the category of all distributive bisemilattices, with all homomorphisms as morphisms. Let $\mathscr{L} N$ be the category of all compact totally disconnected partially ordered left normal bands with constants. The morphisms of $\mathscr{L N}$ are all continuous order preserving band homomorphisms that preserve the constants. The hom-functors on $\mathscr{D} \mathscr{B}$ and $\mathscr{L N}$ are denoted by $\operatorname{Hom}_{b}(-,-)$ and $\operatorname{Hom}_{n}(-,-)$, respectively. We may view 3 either as a distributive bisemilattice or as an $\mathscr{L} \mathscr{N}$-object. If $(S, \cdot,+)$ is a distributive bisemilattice, let $\widehat{S}=\operatorname{Hom}_{b}(S, 3)$. Then $\left(\hat{S}, \star, \leq, c_{0}, c_{1}, c_{\alpha}\right)$ is the partially ordered left normal band with constants of bisemilattice homomorphisms, introduced in 4.17. As a subalgebra of $\left(3^{S}, \star, \leq, c_{0}, c_{1}, c_{\alpha}\right), \widehat{S}$ is 
closed, and hence $\left(\widehat{S}, \star, \leq, c_{0}, c_{1}, c_{\alpha}\right)$ belongs to $\mathscr{L N}$.

Conversely, if $\left(A, \star, \leq, c_{0}, c_{1}, c_{\alpha}, \tau\right)$ is a compact totally disconnected partially ordered left normal band with constants, then define

$$
\widehat{A}=\operatorname{Hom}_{n}(A, 3) \text {. }
$$

Then $(\hat{A}, \cdot,+)$ is a sub-bisemillatice of $\left(\widetilde{A}^{c}, \cdot,+\right)$ as introduced in 5.4. Therefore $\widehat{A}$ is a distributive bisemilattice. (The proof that $\phi+\psi$ and $\phi \cdot \psi$ are continuous whenever $\psi$ and $\phi$ are, is a standard argument.) Hence, if we extend $\operatorname{Hom}_{b}(-, 3)$ and $\operatorname{Hom}_{n}(-, 3)$ in the now expected way to homomorphisms, we obtain contravariant functors

$$
\operatorname{Hom}_{b}(-, 3): \mathscr{D S} \rightarrow \mathscr{L N}, \quad \operatorname{Hom}_{n}(-, 3): \mathscr{L N} \rightarrow \mathscr{D} \mathscr{B} .
$$

As usual, if $S \in \mathscr{D} \mathscr{B}$ and if $A \in \mathscr{L} \mathcal{N}$, we have evaluation maps

given by

$$
\varepsilon_{S}: S \rightarrow \widehat{\widehat{S}}, \quad \delta_{A}: A \rightarrow \widehat{\hat{A}}
$$

$$
\begin{array}{ll}
\phi\left(x \varepsilon_{S}\right)=x \phi & \text { for every } \phi \in \hat{S}, \\
\phi\left(x \delta_{A}\right)=x \phi & \text { for every } \phi \in \widehat{A} .
\end{array}
$$

Standard arguments using the fact that every distributive bisemilattice is a subalgebra of $\left(3^{X}, \cdot,+\right)$ as well as 6.5 yield that $\varepsilon_{S}$ and $\delta_{A}$ are injective and respect the algebraic and topological structure. Our aim is to verify that both maps $\varepsilon_{S}$ and $\delta_{A}$ are surjective. First, we will restrict our attention to the case where $S$ and $A$ are finite, and then use category theoretical methods to handle the general case. An alternative approach would use the idea of "continuous Plonka sums." However, we do not have the tools of "continuous Plonka sums" at this point.

7.1. Proposition. If $(S, \cdot,+)$ is a finite distributive bisemilattice, then $\varepsilon_{S}: S \rightarrow \widehat{\hat{S}}$ is an isomorphism of bisemilattices.

Proof. Let $(S, \cdot,+)$ be a finite distributive bisemilattice, and let $X=$ $\operatorname{Hom}_{b}(S, 3)$ be the set of all homomorphisms from $(S, \cdot,+)$ into $(3, \cdot,+)$. Since $(S, \cdot,+)$ is a subdirect product of $(3, \cdot,+)[15]$, the injectivity of the map $\varepsilon_{S}$ follows.

Now let $\lambda: \widehat{S} \rightarrow \mathbf{3}$ be an order preserving $\star$-homomorphism that preserves the constants. We have to find an element $a \in S$ such that $\phi \lambda=a \phi$ for all $\phi \in \widehat{S}$. Let $N=\{\phi \in \widehat{S}: \phi \lambda \neq \alpha\}$ and let

$$
Z=\{x \in S: x \phi \neq \alpha \text { for all } \phi \in N\} \text {. }
$$

First, we note that $Z \neq \varnothing$. Indeed, let $\left(\phi_{1}, \ldots, \phi_{n}\right)$ be any fixed sequence of all the elements of $N$, and let $\phi_{1} \star \cdots \star \phi_{n}$. Then, since $\lambda$ is a $\star-$ homomorphism, we conclude that $\phi_{N} \in N$, and therefore $\phi_{N} \neq c_{\alpha}$. Hence 
there is an element $x \in S$ such that $x \phi_{N} \neq \alpha$. It follows that $\left(x \phi_{1}\right) \star \cdots \star$ $\left(x \phi_{n}\right) \neq \alpha$ and therefore $x \phi_{i} \neq \alpha$ for all $1 \leq i \leq n$. We conclude that $x \in Z$.

Let $Z=\left\{x_{1}, \ldots, x_{m}\right\}$ and let

$$
e_{Z}=x_{1}+\cdots+x_{m}, \quad n_{Z}=x_{1} \cdots x_{m} .
$$

Note that by [1],

$$
n_{Z}+n_{Z} e_{Z}=n_{Z}, \quad e_{Z}+e_{Z} n_{Z}=e_{Z}
$$

Further, since each $\phi \in N$ is a homomorphism with respect to + and $\cdot$, we conclude that $e_{Z}, n_{Z} \in Z$.

Next, we show

$$
\begin{gathered}
n_{Z} \leq x \text { and } \phi \lambda \neq \alpha \Rightarrow c \phi \neq \alpha, \\
x \leq_{+} e_{Z} \text { and } \phi \lambda \neq \alpha \Rightarrow x \phi \neq \alpha .
\end{gathered}
$$

Indeed, if $e_{Z}+x=e_{Z}$, then $\left(e_{Z}+x\right) \phi=\left(e_{Z} \phi\right)+(x \phi)=e_{Z} \phi$. If $\phi \in N$, then $e_{Z} \phi \neq \alpha$, and therefore $x \phi \neq \alpha$. It follows that the second implication holds. The first implication is proved similarly.

The last implications yield

$$
Z=\left\{x \in S: n_{Z} \leq x\right\}=\left\{x \in S: x \leq_{+} e_{Z}\right\} .
$$

Let $\pi: S \rightarrow I$ be the canonical homomorphism of $(S, \cdot,+)$ onto the bisemilattice replica $(I, \cdot, \cdot)$ of $(S, \cdot,+)$. By the definition of $e_{Z}$ and $n_{Z}$ it is clear that $e_{Z} \pi=n_{Z} \pi$. Let $Z_{0}=\left\{x \in S: x \pi=e_{Z} \pi\right\}$. then $Z_{0} \subseteq Z$. Indeed, since $Z_{0}$ is a distributive lattice, the image of $Z_{0}$ under each bisemilattice homomorphism $\phi: S \rightarrow 3$ is a distributive lattice. Hence we have either $Z_{0} \phi=\{\alpha\}$ or $Z_{0} \phi \subseteq\{0,1\}$. Since $e_{Z} \phi_{N} \neq \alpha$, it follows that $Z_{0} \phi_{N} \subseteq\{0,1\}$, and hence $Z_{0} \subseteq Z=\left\{x \in S: x \phi_{N} \neq \alpha\right\}$.

Note that

$$
Z_{0}=\left\{x \in S: n_{Z} \leq . x \leq . e_{Z}\right\}=\left\{x \in S: n_{Z} \leq_{+} x \leq_{+} e_{Z}\right\} .
$$

Moreover, if $x \in Z$, then $Z_{0} \pi \leq x \pi$, and it follows that $Z_{0}$ is the least Płonka fibre with elements $x$ satisfying $x \phi \neq \alpha$ for all $\phi \in N$.

Furthermore,

$$
x \in Z \Rightarrow\left(n_{Z}+x\right) \cdot e_{Z} \in Z_{0} .
$$

Indeed, if $x \in Z$, then $n_{Z} \cdot x=n_{Z}$ and $\left(n_{Z}+x\right) \pi=\left(n_{Z} \cdot x\right) \pi$, and therefore $\left(\left(n_{Z}+x\right) \cdot e_{Z}\right) \pi=\left(n_{Z} \cdot x \cdot e_{Z}\right) \pi=\left(n_{Z} \cdot e_{Z}\right) \pi=n_{Z} \pi=e_{Z} \pi$.

We also have $N=\left\{\phi \in \widehat{S}: \phi_{N} \star \phi=\phi_{N}\right\}$. Indeed, if $\phi_{N} \star \phi=\phi_{N}$, then $\left(\phi_{N} \lambda\right) \star(\phi \lambda)=\phi_{N} \lambda \neq \alpha$, and hence $\phi \lambda \neq \alpha$. Consequently, $\phi \in N$.

Conversely, if $\phi \in N$, then $\phi=\phi_{i}$ for some $i$, and hence

$$
\phi_{N} \star \phi=\left(\phi_{1} \star \cdots \star \phi_{i} \star \cdots \star \phi_{n}\right) \star \phi_{i}=\phi_{1} \star \cdots \star\left(\phi_{i} \star \phi_{i}\right) \star \cdots \star \phi_{n}=\phi_{N} .
$$


By definition it is clear that $Z=\left\{x \in S: x \phi_{N} \neq \alpha\right\}$. In the following step, we show that

$$
\text { if } x \in Z_{0}, \quad \text { then } \phi \lambda=\alpha \Leftrightarrow x \phi=\alpha \text {. }
$$

Indeed, assume that $x \phi \neq \alpha$. We have to show that $\phi_{N} \star \phi=\phi_{N}$. Assume not. Then we can find an element $y$ such that $\left(y \phi_{N}\right) \star(y \phi) \neq y \phi_{N}$. It follows that $y \phi=\alpha$ and $y \phi_{N} \neq \alpha$. Hence $y \in Z$, from which it follows that $\left(n_{z}+y\right) \cdot e_{Z} \in Z_{0}$. Since $\alpha=\left(\left(n_{Z}+y\right) \cdot e_{Z}\right) \phi$, and since $Z_{0}$ and $Z_{0} \phi$ are distributive lattices, we conclude that $n_{Z} \phi=e_{Z} \phi=\alpha$, and therefore $x \phi=\alpha$, a contradiction. It follows that $\phi \lambda \neq \alpha$.

Conversely, if $\phi \lambda \neq \alpha$, then $\phi \in N$, and therefore $x \phi \neq \alpha$, since $x \in$ $Z_{0} \subseteq Z$.

In order to prepare for the following steps, we need

if $\phi \lambda \neq \alpha$ and $x \phi=0$ for all $x \in Z_{0}$ then $\phi \star \phi_{N}=c_{0} \star \phi_{N}$.

Indeed, $x \phi=0$ for all $x \in Z_{0}$ implies $e_{Z} \phi=0$. If $x \in Z$ is given, then $x \leq_{+} e_{Z}$, and hence $x \phi \leq_{+} 0$ that is, $x \phi=0$. We conclude that $Z \phi=\{0\}$. Hence,

$$
\begin{aligned}
x\left(\phi \star \phi_{N}\right) & =(x \phi) \star\left(x \phi_{N}\right) \\
& = \begin{cases}0 \star\left(x \phi_{N}\right) & \text { if } x \in Z, \\
x \phi \star \alpha & \text { if } x \notin Z,\end{cases} \\
& = \begin{cases}0 & \text { if } x \in Z, \\
\alpha & \text { if } x \notin Z,\end{cases} \\
& =\left(x c_{0}\right) \star\left(x \phi_{N}\right)=x\left(c_{0} \star \phi_{N}\right) .
\end{aligned}
$$

Further,

if $\phi \lambda \neq \alpha$ and $x \phi=0$ for each $x \in Z_{0}$ then $\phi \lambda=0$.

Let us compute: $\phi_{N} \lambda \neq \alpha$ implies

$$
\phi \lambda=(\phi \lambda) \star\left(\phi_{N} \lambda\right)=\left(\phi \star \phi_{N}\right) \lambda=\left(c_{0} \star \phi_{N}\right) \lambda=\left(c_{0} \lambda\right) \star\left(\phi_{N}\right) \lambda=0 .
$$

Next, for every $\phi \in N$ with $e_{Z} \phi=1$ let

$$
a_{\phi}=\prod\left\{x \in Z_{0}: x \phi=1\right\} \text {. }
$$

First, note that by our previous statement, $\phi \lambda=1$ implies that there is at least one $x \in Z_{0}$ such that $x \phi=1$, and hence $e_{Z} \phi=1$. Therefore, $a_{\phi}$ is defined for all $\phi$ with $\phi \lambda=1$. Also, since $\phi: Z_{0} \rightarrow\{0,1\}$ is a lattice homomorphism, $a_{\phi}$ is a co-prime (that is, join irreducible) element of the distributive lattice $Z_{0}$. Moreover,

$$
\text { if } e_{Z} \phi=1=e_{Z} \psi \text {, then } a_{\phi} \leq a_{\pi} \Leftrightarrow \psi \star \phi_{N} \leq \phi \star \phi_{N} \text {. }
$$

For a proof of this statement, note that $x \in Z_{0}$ implies $x \phi_{N} \neq \alpha$ and therefore $x\left(\phi \star \phi_{N}\right)=x \phi$. Hence $\psi \star \phi_{N} \leq \phi \star \phi_{N}$ implies $a_{\phi} \leq a_{\psi}$. 
Conversely, assume that $a_{\phi} \leq a_{\psi}$. Note that if $x\left(\psi \star \phi_{N}\right) \neq \alpha$, then $x \phi_{N} \neq \alpha$, and hence $x \in Z$. Thus, if $x\left(\psi \star \phi_{N}\right)=1$ then $e_{Z}\left(\psi \star \phi_{N}\right)=1$ and hence $\left(\left(n_{Z}+x\right) \cdot e_{Z}\right)\left(\psi \star \phi_{N}\right)=1$. Since $\left(n_{Z}+x\right) \cdot e_{Z} \in Z_{0}$, we conclude that $a_{\phi} \leq a_{\psi} \leq\left(n_{Z}+x\right) \cdot e_{Z}$, and hence $\left(\left(n_{Z}+x\right) \cdot e_{Z}\right) \phi=1$. If $n_{Z} \phi=0$, then $x \phi=1$ and therefore $x\left(\phi \star \phi_{N}\right)=1$. If, however, $n_{Z} \phi=1$, then $n_{Z} \leq x$ implies $x \phi=1$, and therefore $x\left(\phi \star \phi_{N}\right)=1$ in this case, too. Secondly, if $x\left(\psi \star \phi_{N}\right)=0$, then $x \in Z$, and, since $\phi \star \phi_{N} \in N$, we have $x\left(\phi \star \phi_{N}\right) \in\{0,1\}$. These two cases together imply $\psi \star \phi_{N} \leq \phi \star \phi_{N}$.

Finally, let

$$
a_{\lambda}=\sum\left\{a_{\phi}: \phi \lambda=1\right\}
$$

Then we would like to show that $\phi \lambda=a_{\lambda} \phi$ for all $\phi \in \widehat{S}$. Since $a_{\lambda} \in Z_{0}$, this equation is at least true for those $\phi$ 's for which $\phi \lambda=\alpha$ or for which $a_{\lambda} \phi=\alpha$. Hence it remains to show that

$$
\phi \lambda=1 \Leftrightarrow a_{\lambda} \phi=1 .
$$

Assume that $\phi \lambda=1$. Then we obtain $a_{\phi} \leq_{+} a_{\lambda}$, and therefore

$$
1=a_{\phi} \phi \leq_{+} a_{\lambda} \phi \neq \alpha,
$$

that is, $a_{\lambda} \phi=1$. Conversely, assume that $a_{\lambda} \phi=1$. Then, by definition, $a_{\phi} \leq a_{\lambda}$. Since $Z_{0}$ is a distributive lattice, we also have $a_{\phi} \leq_{+} a_{\lambda}$, and since $a_{\phi}$ is co-prime in $Z_{0}$, there is an element $\psi \in \widehat{S}$ such that $\psi \lambda=1$ and $a_{\phi} \leq a_{\psi}$. We have shown that this implies $\psi \star \phi_{N} \leq \phi \star \phi_{N}$, and we arrive at

$$
1=\psi \lambda=\left(\psi \star \phi_{N}\right) \lambda \leq\left(\phi \star \phi_{N}\right) \lambda=\phi \lambda .
$$

This completes the proof of 7.1 .

In the next step, we have to start with a finite partially ordered left normal band with constants, say $\left(A, \star, \leq, c_{0}, c_{1}, c_{\alpha}\right)$, and we have to show that the map $\delta_{A}: A \rightarrow \widehat{\hat{A}}$ is surjective. The arguments are very similar.

7.2. Proposition. If $\left(A, \star, \leq, c_{0}, c_{1}, c_{\alpha}\right)$ is a finite partially ordered left normal band with constants, then $\delta_{A}: A \rightarrow \widehat{\hat{A}}$ is an isomorphism of partially ordered left normal bands with constants.

Proof. The injectivity of $\delta_{A}$ follows from 4.15. In order to show the surjectivity of $\delta_{A}$, we start with a bisemilattice homomorphism $\lambda: \hat{A} \rightarrow 3$. We have to find an element $a_{\lambda} \in A$ so that $a_{\lambda} \phi=\phi \lambda$ for all $\phi \in \hat{A}$. So, let $N=\{\phi \in \widehat{A}: \phi \lambda \neq \alpha\}$ and let

$$
Z=\{x \in A: x \phi \neq \alpha \text { for all } \phi \in N\} .
$$


First, we note that $Z \neq \varnothing$. Indeed, $c_{0}, c_{1} \in Z$. If $N=\left\{\phi_{1}, \ldots, \phi_{n}\right\}$, let

$$
\alpha_{N}=\phi_{1} \cdot \ldots \cdot \phi_{n}, \quad \omega_{N}=\phi_{1}+\cdots+\phi_{n} .
$$

Then, since $\lambda$ is a bisemilattice homomorphism, we conclude that $\alpha_{N}, \omega_{N} \in$ $N$. Let $\left(x_{1}, \ldots, x_{m}\right)$ be any fixed sequence of all elements of $Z$, and we arrange this sequence such that $x_{1}=c_{1}$. Let

$$
n_{z}=x_{1} \star \cdots \star x_{m} \text {. }
$$

Then, since each $\phi \in N$ is a band homomorphism, we conclude that $n_{Z} \in Z$, indeed $n_{Z} \phi=1$ for all $\phi \in N$. From the definitions it follows that

$$
N=\left\{\phi \in \widehat{A}: \alpha_{N} \leq \phi\right\}=\left\{\phi \in \widehat{A}: \phi \leq_{+} \omega_{N}\right\}
$$

and

$$
Z=\left\{x \in A: n_{z} \star x=n_{Z}\right\} .
$$

By definition it is clear that

$$
Z=\left\{x \in A: x \alpha_{N} \neq \alpha\right\}=\left\{x \in A: x \omega_{N} \neq \alpha\right\} .
$$

Let $\pi: A \rightarrow I$ be the canonical homomorphism of $(A, \star)$ onto the semilattice replica $(I, \star)$ of $(A, \star)$. Let $Z_{0}=\left\{x \in A: x \pi=n_{Z} \pi\right\}$. Then

$$
Z_{0}=\left\{x \in A: x=x \star n_{Z}, n_{Z}=n_{Z} \star x\right\},
$$

and therefore $Z_{0} \subseteq Z$. Furthermore;

$$
x \in Z \Rightarrow x \star n_{Z} \in Z_{0} \text {. }
$$

Indeed, if $x \in Z$, then $n_{Z} \star x=n_{Z}$ and therefore $n_{Z} \star\left(x \star n_{Z}\right)=\left(n_{Z} \star x\right) \star$ $n_{Z}=n_{Z}$ and $\left(x \star n_{Z}\right) \star n_{Z}=x \star\left(n_{Z} \star n_{Z}\right)=x \star n_{Z}$.

Next, we show $N=\left\{\phi \in \hat{A}: n_{Z} \phi \neq \alpha\right\}$. If $n_{Z} \phi \neq \alpha$, then $x \phi \neq \alpha$ whenever $x \in Z$, hence $\left\{x \in A: x \alpha_{N} \neq \alpha\right\}=Z \subseteq\{x \in A: x \phi \neq \alpha\}$ and thus $\{x: x \phi=\alpha\} \subseteq\left\{x: x \alpha_{N}=\alpha\right\}$. It follows that $x \alpha_{N}+x \alpha_{N} \cdot x \phi=x \alpha_{N}$ for all $x \in A$, that is, $\alpha_{N}+\alpha_{N} \cdot \phi=\alpha_{N}$. We conclude that $\alpha_{N} \lambda+\alpha_{N} \lambda \cdot \phi \lambda=\alpha_{N} \lambda$. Since $\alpha_{N} \lambda \neq \alpha$, this can happen only if $\phi \lambda \neq \alpha$, that is, only if $\phi \in N$. The other direction follows easily from the definitions.

Moreover,

$$
y \in Z \text { and } y \alpha_{N}=1 \Leftrightarrow y \star n_{Z}=c_{1} \star n_{Z} .
$$

If $y \star n_{Z}=c_{1} \star n_{Z}$, then, since $n_{Z} \alpha_{N} \neq \alpha$,

$$
1=c_{1} \alpha_{N}=\left(c_{1} \star n_{Z}\right) \alpha_{N}=\left(y \star n_{Z}\right) \alpha_{N}=y \alpha_{N} .
$$

Conversely, assume that $y \alpha_{N}+1$. since $y \leq c_{1}$, it follows that $y \star n_{Z} \leq$ $c_{1} \star n_{Z}$. If $y \star n_{Z} \neq c_{1} \star n_{Z}$, then we could find an element $\phi \in \hat{A}$ such that $\left(y \star n_{Z}\right) \phi<\left(c_{1} \star n_{Z}\right) \phi$. Now $y \star n_{Z}, c_{1} \star n_{z} \in Z_{0}$, and the image of $Z_{0}$ is a partially ordered left-zero band with more than one element, 
contained in 3. Hence $\left(y \star n_{Z}\right) \phi=0$ and $\left(c_{1} \star n_{z}\right) \phi=1$. It follows that $c_{1} \phi \star n_{Z} \phi=1$ and hence $n_{Z} \phi \neq \alpha$. Therefore, since $\phi \in N$, we have $\alpha_{N} \leq \phi$, and this implies that $1=y \alpha_{N} \leq y \phi$. Consequently, $y \phi=1$, and hence $0=\left(y \star n_{Z}\right) \phi=y \phi \star n_{Z} \phi=1$, a contradiction.

In the following step, we show that

$$
\text { if } x \in Z_{0} \text { then } \phi \lambda=\alpha \Leftrightarrow x \phi=\alpha \text {. }
$$

Indeed, fix an $x \in Z_{0}$ and assume that $\phi \lambda \neq \alpha$. Then $\phi \in N$, and since $x \in Z_{0} \subseteq Z, x \phi \neq \alpha$. Conversely, let $x \phi \neq \alpha$. We have to show that $\phi \lambda \neq \alpha$, that is, $\phi \in N$. But this is equivalent to showing that $n_{Z} \phi \neq \alpha$. Since the image of $Z_{0}$ under $\phi$ is a partially ordered left-zero band, we have either $Z_{0} \phi \subseteq\{0,1\}$ or $Z_{0} \phi=\{\alpha\}$. Since $x \in Z_{0}$ and $x \phi \neq \alpha$, the second case is impossible, and we obtain $n_{z} \phi \neq \alpha$.

For every $\phi \in N$ let $F_{\phi}=\left\{x \in Z_{0}: x \phi=1\right\}$. Then $n_{z} \in F_{\phi}$ for all $\phi \in N$, and

$$
\begin{gathered}
F_{\phi+\psi}=F_{\phi} \cup F_{\psi}, \quad F_{\phi \cdot \psi}=F_{\phi} \cap F_{\psi}, \\
F_{\phi} \subseteq F_{\psi} \Leftrightarrow \phi+\phi \cdot \alpha_{N} \leq \psi+\psi \cdot \alpha_{N} .
\end{gathered}
$$

Only the last statement needs proving. If $\phi+\phi \cdot \alpha_{N} \leq \psi+\psi \cdot \alpha_{N}$, then, since $x \alpha_{N} \neq \alpha$ for each $x \in Z_{0}$ and since $\alpha_{N} \leq \phi$ implies $\phi+\phi \cdot \alpha_{N}=\phi$, we obtain $x \phi=x\left(\phi+\phi \cdot \alpha_{N}\right) \leq x\left(\psi+\psi \cdot \alpha_{N}\right)=x \psi$ for each $x \in Z_{0}$, and hence $F_{\phi} \subseteq F_{\psi}$. Conversely, assume that $F_{\phi} \subseteq F_{\psi}$. If $\phi+\phi \cdot \alpha_{N} \not$. $\psi+\psi \cdot \alpha_{N}$, then we could find an element $y \in A$ so that $y\left(\phi+\phi \cdot \alpha_{N}\right) \not . y\left(\psi+\psi \cdot \alpha_{N}\right)$. This can only happen if $y \alpha_{N} \neq \alpha$, and therefore $y \in Z$. Hence it would follow that $y \phi, y \psi \in\{0,1\}$ and $y \phi \not . y \psi$, that is, $y \phi=1, y \psi=0$. Let $x=y \star n_{Z} \in Z_{0}$. Then, since $n_{Z} \phi \neq \alpha \neq n_{Z} \psi$, we obtain $x \phi=\left(y \star n_{Z}\right) \phi=$ $y \phi=1$ and $x \psi=0$, a contradiction to $F_{\phi} \subseteq F_{\psi}$.

Let $F_{\lambda}=\bigcap\left\{F_{\phi}: \phi \lambda=1\right\}$. Note that $F_{\lambda}=F_{\phi_{0}}$ for a certain $\phi_{0}$ with $\phi_{0} \lambda=1$. Then

$$
\phi \lambda=1 \Leftrightarrow F_{\lambda} \subseteq F_{\phi} .
$$

Clearly, by definition, $\phi \lambda=1$ implies $F_{\lambda} \subseteq F_{\phi}$. Conversely, as $F_{\lambda} \subseteq F_{\phi}$. Then $\phi_{0}+\phi_{0} \cdot \alpha_{N} \leq \phi+\phi \cdot \alpha_{N}$, and hence $1=\phi_{0} \lambda=\left(\phi_{0}+\phi_{0} \cdot \alpha_{N}\right) \lambda \leq$. $\left(\phi+\phi \cdot \alpha_{N}\right) \lambda=\phi \lambda$, that is, $\phi \lambda=1$.

We would like to show that $F_{\lambda}$ has a unique minimal element. Let $f_{1}, \ldots, f_{m}$ be the minimal elements of $F_{\lambda}$, and suppose that there is more than one. For each $i$ find $\phi_{i} \in \hat{A}$ so that $f_{i} \phi_{i}=1$, but $f_{j} \phi_{i}=0$ for some $j \neq i$. The each $\phi_{i}$ maps $Z_{0}$ into $\{0,1\}$, and hence all the $\phi$ 's belong to $N$. Let $\phi=\phi_{1}+\cdots+\phi_{m}$. It follows that $F_{\phi}$ contains all minimal elements of $F_{\lambda}$, and therefore $F_{\lambda} \subseteq F_{\phi}$. Hence $\left(\phi_{1}+\cdots+\phi_{m}\right) \lambda=1$, which implies that $\phi_{i} \lambda=1$ for at least one $i$. For this $i$ we would have $F_{\lambda} \subseteq F_{\phi_{i}}$, a contradiction to the choice of $\phi_{i}$. 
Let $a_{\lambda}$ be the unique minimal element of $F_{\lambda}$. Then by our previous results

$$
a_{\lambda} \phi=\alpha \Leftrightarrow \phi \lambda=\alpha, \quad a_{\lambda} \phi=1 \Leftrightarrow \phi \lambda=1
$$

and hence $a_{\lambda} \phi=\phi \lambda$ for all $\phi \in \widehat{A}$.

We now show that $\varepsilon_{S}$ and $\delta_{A}$ are always bijections. We need the following propositions:

7.3. Proposition. The functor $\operatorname{Hom}_{b}(-, 3): \mathscr{D} \mathscr{B} \rightarrow \mathscr{L N}$ carries limits of directed systems onto colimits of (co-) directed systems, and the functor $\operatorname{Hom}_{n}(-, 3): \mathscr{L N} \rightarrow \mathscr{D} \mathscr{B}$ carries colimits of (co-) directed systems of finite $\mathscr{L} \mathbb{N}$-objects onto limits of directed systems.

Proof. The first assertion follows from a standard property of the contravariant hom-functor. For the second property, let $A \in \mathscr{L N}$ be a colimit of a (co-)directed system of finite objects $A_{i} \in \mathscr{L N}, i \in I$, where $I$ is a directed set. For each $i<j$ let $\rho_{j, i}: A_{j} \rightarrow A_{i}$ be the map defining the directed system, and for each $i \in I$ let $\rho_{i}: A \rightarrow A_{i}$ be the canonical map. Then we have the following representation of $A$ :

$$
A=\left\{x \in \prod_{i \in I} A_{i}: x_{j} \rho_{j, i}=x_{i} \text { for all } i<j\right\} .
$$

Let

$$
\operatorname{ker} \rho_{i}=\left\{(x, y) \in A: x \rho_{i}=y \rho_{i}\right\}
$$

It follows that

$$
\bigcap_{i \in I} \operatorname{ker} \rho_{\Delta}=\{(x, x): x \in A\}
$$

and that

$$
i \leq j \Rightarrow \operatorname{ker} \rho_{j} \subseteq \operatorname{ker} \rho_{i} .
$$

Moreover, since each $A_{i}$ is finite, all the ker $\rho_{i}$ 's are open and closed subsets of $A \times A$.

In order to show that $\operatorname{Hom}_{n}(-, 3)$ maps colimits to limits, it suffices to show that for every continuous order preserving band homomorphism 
$\phi: A \rightarrow 3$ there is an index $i \in I$ and an order preserving band homomorphism $\phi_{i}: A \rightarrow 3$ such that $\phi=\rho_{i} \circ \phi_{i}$. Let $\operatorname{ker} \phi=\{(x, y) \in A: x \phi=y \phi\}$. Then the existence of $i \in I$ and $\phi_{i}: A_{i} \rightarrow 3$ with $\phi=\rho_{i} \circ \phi_{i}$ is equivalent with

$$
(\exists i \in I) \quad \operatorname{ker} \rho_{i} \subseteq \operatorname{ker} \phi .
$$

Now note that $\operatorname{ker} \phi \subseteq A \times A$ is open and closed, and $\bigcap \operatorname{ker} \rho_{i}=\Delta \subseteq \phi$. Since all the sets $\operatorname{ker} \rho_{i}$ are closed, compactness of $A \times A$ yields the existence of finitely many indices $i_{1}, \ldots, i_{n} \in I$ such that

$$
\operatorname{ker} \rho_{i_{1}} \cap \cdots \cap \operatorname{ker} \rho_{i_{n}} \subseteq \operatorname{ker} \phi .
$$

Since $I$ is directed, we can find an index $i_{0} \in I$ such that $i_{0} \geq i_{1}, \ldots, i_{n}$, and for this particular $i_{0}$ we obtain $\operatorname{ker} \rho_{i_{0}} \subseteq \operatorname{ker} \phi$.

7.4. Proposition. Every distributive bisemilattice $S \in \mathscr{D} \mathscr{B}$ is a directed limit of finite distributive bisemilattices, and every compact, totally disconnected partially ordered left normal band with constants $A \in \mathscr{L} N$ is a codirected colimited of finite partially ordered left normal bands with constants.

Proof. The first assertion follows from the fact that finitely generated distributive bisemilattices are finite, and the second claim follows from the observation that for every $A \in \mathscr{L N}$ the $\mathscr{L N}$-morphisms from $A$ into finite partially ordered left normal bands separate the points of $A$ by 6.5 .

Now an application of 7.1 through 7.4 yields

7.5. THEOREM. The categories $\mathscr{D} \mathscr{B}$ and $\mathscr{L N}$ are dual to each other under the functors $\operatorname{Hom}_{b}(-, 3): \mathscr{D \mathscr { B }} \rightarrow \mathscr{L N}$ and $\operatorname{Hom}_{n}(-, 3): \mathscr{L N} \rightarrow \mathscr{D \mathscr { B }}$.

\section{References}

[1] R. Balbes, 'A representation theorem for distributive quasi-lattices', Find. Math. 68 (1970), 207-214.

[2] G. Birkhoff, Lattice theory, Colloquium Publications, vol. 25, Amer. Math. Soc., Providence, R.I., 3rd ed., 1967.

[3] B. A. Davey, 'Topological duality for prevarieties of universal algebras', in Studies in Foundations and Combinatorics, G. Rota, ed., Academic Press, New York, London, 1978, 61-98.

[4] L. Fuchs, Partially ordered algebraic systems, Pergamon Press, 1963.

[5] J. Gerhardt, 'Subdirectly irreducible idempotent semigroups', Pacific J. Math. 39 (1971), 669-676. 
[6] K. Halkowska, 'O pewnej równosciowo definiowalnej klasie algebr', Zeszyty Naukowe W.S.P., Opole, Ser. Matematyka 19 (1976), 127-136.

[7] K. Hofmann and K. Keimel, A general character theory for partially ordered sets and lattices, vol. 122, Mem. Amer. Math. Soc., 1972.

[8] K. Hofmann, M. Mislov, and A. Stralka, The Pontrjagin duality for semilattices, Lecture Notes in Mathematics, vol. 396, Springer-Verlag, Berlin, Heidelberg, New York, 1974.

[9] J. Kalman, 'Subdirect decomposition of distributive quasilattices', Fund. Math. 71 (1971), 161-163.

[10.] W. Kimura and M. Yamada, 'Note on idempotent semigroups II', Proc. Japan. Acad. 34 (1958), 110-112.

[11] H. Lakser, R. Padmanabhan, and C. Platt, 'Subdirect decompositions of Płonka sums', Duke Math. J. 39 (1972), 485-488.

[12] S. Mac Lane, Categories for the Working Mathematician, Graduate Texts in Mathematics, vol. 5, Springer-Verlag, 1971.

[13] L. Nachbin, Topology and Order, Van Nostrand, 1965.

[14] J. Płonka, 'On a method of construction of abstract algebras', Fund. Math. 61 (1967), 183-189.

[15] J. Plonka, ‘On distributive quasilattices', Fund. Math. 60 (1967), 191-200.

[16] J. Płonka, 'Some remarks on sums of direct systems of algebras', Fund. Math. 62 (1968), 301-308.

[17] R. Padmanabhan, 'Regular identities in lattices', Trans. Amer. Math. Soc. 158 (1971), 179-189.

[18] H. Priestley, 'Representation of distributive lattices by means of ordered Stone spaces', Bull. London Math. Soc. 2 (1970), 186-190.

[19] H. Priestley, 'Ordered topological spaces and the representation of distributive lattices', Proc. London Math. Soc. 3 (1972), 506-530.

[20] A. Romanowska, 'Constructing and reconstructing of algebras', Demonstratio Math. 18 (1985), 209-230.

[21] A. Romanowska and J. Smith, 'Distributive lattices, generalizations and related nonassociative structures', Houston J. Math. 11 (1985), 367-386.

[22] A. Romanowska and J. Smith, Modal Theory-An Algebraic Approach to Order, Geometry and Convexity, Heldermann-Verlag, Berlin, 1985.

[23] A. Romanowska and J. Smith, 'On the structure of semilattice sums', preprint, 1987.

[24] M. Stone, 'Topological representations for Boolean algebras', Trans. Amer. Math. Soc. 40 (1936), 37-111.

[25] M. Stone, 'Topological representations for distributive lattices and brouwerian logics', Casopis Pěst. Mat. 67 (1937), 1-25.

Department of Mathematics

University of California

Riverside, California 92521

U.S.A.
Institute of Mathematics Warsaw Technical University

00661 Warsaw

Poland 